\title{
Prevalência e Fatores Associados ao Consumo de Álcool e Tabaco entre Estudantes de Medicina no Nordeste do Brasil
}

\section{Prevalence and Associated Factors of Alcohol Consumption and Smoking among Medical Students in Northeastern Brazil}

\author{
Marcelo de Almeida Pinheiro \\ Levi Freitas Torres ${ }^{i}$ \\ Matheus Sales Bezerral \\ Rodrigo Cardoso Cavalcante ${ }^{I}$ \\ Raquel Diógenes Alencarll \\ Amanda Carneiro Donato ${ }^{I I I}$ \\ Camila Pontes Bessa Campêlo ${ }^{I V}$ \\ Ileana Pitombeira Gomes ${ }^{I I}$ \\ Carlos Henrique Alencar ${ }^{I I}$ \\ Luciano Pamplona de Góes Cavalcantill
}

\section{PALAVRAS-CHAVE \\ - Hábito de Fumar. \\ - Alcoolismo. \\ - Estudantes. \\ - Educação de Graduação em Medicina.}

REVISTA BRASILEIRA DE EDUCAÇÃO MÉDICA

\begin{abstract}
RESUMO
Introdução: $O$ consumo de derivados de tabaco e álcool é apontado como importante causa de doenças e agravos no mundo. No Brasil, há um aumento no consumo dessas drogas entre os jovens, principalmente estudantes universitários. Objetivo: Conhecer a prevalência e os fatores associados ao tabagismo e consumo de bebidas alcoólicas entre estudantes de Medicina, além do nível de conhecimento acerca das técnicas de cessação do hábito tabagista em diferentes momentos da vida acadêmica. Métodos: Estudo analítico, de prevalência, envolvendo estudantes de Medicina de Fortaleza, Ceará, Brasil. Foram selecionadas todas as escolas médicas e os estudantes do primeiro ano (S1/S2), quarto ano (S7/S8) e aqueles do último ano do internato (I3/I4). A amostra foi calculada considerando uma frequência esperada de 10\% de pessoas fumantes, com um erro de 3\%, estimando 726 estudantes das quatro instituições. Foi aplicado um questionário estruturado, com 46 perguntas. Os dados foram analisados pelo software Stata 11.2. Resultados: Foram entrevistados 1.035 estudantes, distribuídos proporcionalmente nos três períodos, $392(37,9 \%)$ do primeiro ano (S1-S2), 319 (30,8\%) do quarto ano (S7-S8) e 324 (31,3\%) do internato (I3-I4). Quinhentos e cinquenta e três (53,4\%) eram do sexo feminino, a maioria era solteira (993; 96,3\%), nascidos em Fortaleza (748; $72,4 \%)$, residiam com os pais $(896 ; 86,8 \%)$ e com renda familiar acima de dez salários mínimos (652; 61,8\%). Ao todo, 533 (51,5\%) eram alunos de instituições particulares. Do total, 254 (24,6\%) já haviam fumado. Esse consumo foi significativamente maior entre o sexo masculino $(p=0,025)$, sem diferença em relação ao estado civil $(p=0,247)$ ou renda familiar $(p=0,191)$. Todos os acadêmicos que experimentaram alguma substância derivada do tabaco já haviam ingerido bebida alcoólica alguma vez na vida $(p<0,000)$. O consumo de álcool foi referido por mais de $80 \%$ dos estudantes, sendo maior entre aqueles cuja família apresentou renda superior a nove salários mínimos ( $p=0,001$ ). Houve relato de embriaguez em mais de $70 \%$ dos estudantes, tendo esse fato ocorrido antes dos 18 anos. Cerveja e vodca são as bebidas mais consumidas. Apenas 39,5\% afirmaram estar aptos a aconselhar um paciente a não ingerir bebidas alcoólicas e apenas $28,4 \%$ receberam algum treinamento sobre o assunto em sua universidade. Conclusão: A prevalência do consumo de álcool é muito elevada entre os estudantes de Medicina, principalmente entre aqueles que relataram fumar. Esses temas são abordados de forma incipiente em sua formação. É preciso reforçar esses aspectos na formação desses futuros profissionais de saúde.
\end{abstract}

\footnotetext{
${ }^{I}$ Centro Universitário Christus, Fortaleza, CE, Brasil.

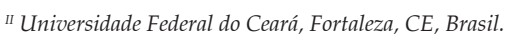

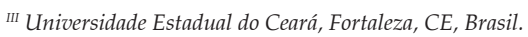

${ }^{I V}$ Universidade de Fortaleza, Fortaleza, CE, Brasil.
} 


\section{KEYWORDS}

- Smoking.

- Alcoholism.

- Students.

- Education Medical Graduate.

Recebido em: 04/07/2016

Aprovado em: 12/02/2017

\section{ABSTRACT}

Introduction: Tobacco and alcohol consumption is considered a major cause of diseases and disorders in the world. In Brazil, there has been increased consumption of these drugs among young people, especially university students. Objective: To discover the prevalence of and factors associated to smoking and alcohol consumption among medical students, as well as their level of knowledge about techniques to stop smoking at different times of their academic life. Methods: Analytical study of prevalence among medical students in Fortaleza, Ceará, Brazil. The study sample included all the city's medical schools and their first year (S1/ S2) and fourth year (S7/S8) students and students in the final year of their internship (I3/I4). The sample was calculated considering an expected smoker frequency of $10 \%$, with a 3\% margin of error, estimating 726 students in the four institutions. A structured questionnaire containing 46 questions was applied. Data were analyzed using Stata 11.2 software. Results: 1,035 students were interviewed, distributed proportionally in the three periods: 392 (37.87\%) from the first year (S1 / S2), 319 (30.82\%) from the fourth year (S7/S8) and 324 (31.30\%) interns (I3/I4). 553 students (53.4\%) were female; most of the students were single (993; 96.3\%), born in Fortaleza $(748 ; 72.4 \%)$, living with their parents $(896 ; 86.8 \%$ ) and with a household income of more than 10 minimum wages (652; 61.8\%). In total, 533 (51.5\%) were students at private institutions. Of the total, 254 (24.6\%) had smoked. This consumption was significantly higher among males ( $p=0.025)$, with no difference in relation to marital status $(p=0.247)$ or household income ( $p$ $=0.191$ ). All the students who reported having experienced any tobacco derivative also reported using alcohol in their lifetime $(p<0.000)$. Alcohol consumption was reported by more than $80 \%$ of the students, and was higher among those whose family income was more than nine times the minimum wage $(p=0.001)$. Alcoholic intoxication was reported by over $70 \%$ of the students - where this had occurred before the age of 18 years. Beer and vodka are the most consumed beverages. Only 39.5\% said they were inclined to advise a patient to avoid alcoholic beverages and only $28.4 \%$ had received training on the subject at their university. Conclusion: The prevalence of alcohol consumption is very high among medical students, especially among those who reported smoking. These issues are addressed in a primitive manner in their training. We must strengthen these aspects in the training of future health professionals.

\section{INTRODUÇÃO}

O consumo de derivados do tabaco e de álcool, há bastante tempo, é apontado como importante causa de doenças e agravos no mundo ${ }^{1,2}$. Em algumas situações, é identificado como a principal razão de alguns tipos de câncer como fator isolado e ainda relacionado diretamente com óbitos por doença pulmonar obstrutiva crônica (DPOC) e doenças cardiovasculares (DCV) $)^{3-5}$.

Há um aumento no consumo de drogas entre os jovens e a experimentação tanto de produtos derivados do tabaco como de álcool, o que eleva o risco de que adultos fumem e façam uso de bebidas alcoólicas, levando a Organização Mundial da Saúde (OMS) a manifestar grande preocupação em evitar a experimentação ou pelo menos adiar esse fato ${ }^{6-10}$. Anualmente, cerca de cinco milhões de pessoas morrem de doenças atribuídas ao cigarro, mesmo sendo o tabagismo a principal causa evitável de morte e de doenças crônicas ${ }^{11}$.

No Brasil, a idade de risco se aproxima daquela em que os estudantes ingressam no ensino superior e há evidências de que o consumo de álcool entre estudantes universitários é maior que na população geral, independentemente do curso ${ }^{7-10}$.
A cidade de Fortaleza, no Nordeste brasileiro, apresenta elevadas taxas de consumo de bebidas alcoólicas, ocupando a segunda posição entre as capitais nordestinas e a terceira em todo o Brasil ${ }^{12}$.

Os estudantes da área da saúde, principalmente os de Medicina, têm sido alvo de programas de orientação e prevenção do consumo de tabaco e bebidas alcoólicas pelo papel social que desempenharão na orientação de comunidades. Entre suas principais atividades, destacam-se educação em saúde, apoiar políticas antitabagistas e influenciar os esforços de controle nacional e mundial do uso do tabaco e do consumo de bebidas alcoólicas ${ }^{6,13,14}$. Entretanto, a educação médica vem sendo subutilizada, evidenciando que os estudantes de Medicina têm recebido treinamento inadequado sobre o tabagismo, bem como sobre o consumo de bebidas alcoólicas e sua dependência ${ }^{15,16}$

Desta forma, o objetivo deste trabalho foi avaliar a prevalência e possíveis fatores associados ao tabagismo e consumo de bebidas alcoólicas entre estudantes de Medicina e o nível de conhecimento acerca das técnicas de cessação do hábito ta- 
bagista e do consumo de álcool em diferentes momentos de sua vida acadêmica.

\section{MÉTODOS}

\section{Desenho do estudo}

Foi realizado um estudo analítico, de prevalência, envolvendo estudantes de Medicina de Fortaleza, Ceará, Brasil. Foram selecionadas as quatro escolas médicas de Fortaleza - duas públicas: Universidade Federal do Ceará (UFC) e Universidade Estadual do Ceará (Uece) e; duas particulares: Universidade de Fortaleza (Unifor) e Centro Universitário Christus (Unichristus). Foram incluídos estudantes de Medicina regularmente matriculados nessas instituições e que cursavam o primeiro ano do curso (S1/S2), o quarto ano do curso (S7/S8) e aqueles do último ano do internato (I3/I4).

\section{Amostra}

Foi considerado como população do estudo o número máximo de alunos nos seis semestres avaliados. Utilizou-se como parâmetro uma frequência esperada de $10 \%$ de pessoas fumantes, com um erro de 3\%. Aos valores calculados foram acrescentados 10\% como correção para possíveis perdas durante a coleta dos dados. Desta forma, a amostra mínima foi estimada em 726 estudantes das quatro instituições.

\section{Coleta de dados}

Foi aplicado um questionário estruturado, contendo 46 perguntas. Os itens referiam-se às características sociodemográficas, informações sobre o consumo de tabaco e ingestão de álcool e sobre como esses temas foram abordados na grade curricular das instituições.

\section{Análise dos dados}

Os dados foram digitados utilizando-se o programa Epi-info Versão 3.5.1, e a análise foi realizada por meio do programa Stata 11.2. Foram utilizados testes paramétricos (este $t$ de Student) ou não paramétricos (chi quadrado de Pearson; teste de Kruskal-Wallis) de acordo com as características das variáveis do estudo. Foram calculadas também as razões de prevalência, bem como seus respectivos intervalos de confiança.

\section{Aspectos éticos}

Este estudo seguiu os princípios éticos de pesquisa envolvendo seres humanos da Resolução 466/12 do Conselho Nacional de Saúde, respeitando os princípios fundamentais de autonomia, beneficência, não maleficência, justiça e equidade. $\mathrm{O}$ estudo foi aprovado pelo Comitê de Ética em Pesquisa (CEP) da Unichristus (Protocolo no 020/2012) e autorizado pelas outras instituições após parecer do CEP.

\section{RESULTADOS}

Foram entrevistados 1.035 estudantes, distribuídos proporcionalmente nos três períodos, $392(37,8 \%)$ do primeiro ano (S1S2), 319 (30,8\%) do quarto ano (S7-S8) e 324 (31,3\%) do internato (I3-I4). Observou-se que $553(53,4 \%)$ eram do sexo feminino, a maioria era solteira (993; 96,3\%), nascidos em Fortaleza (748; $72,4 \%)$, residiam com os pais $(896 ; 86,8 \%)$ e com renda familiar acima de dez salários mínimos (652; 61,8\%). Ao todo, 533 (51,5\%) eram alunos de instituições particulares e 502 (48,5\%) de públicas.

Do total, 254 (24,6\%) já haviam fumado alguma vez na vida. O motivo para ter começado a fumar foi respondido por 191 alunos e 96 destes (50,0\%) afirmaram ter iniciado por vontade própria. Por outro lado, 72 (37,5\%) por influência de amigos ou família, uma vez que 15,2\% apresentam algum familiar que fuma em sua residência.

Ao se estratificarem as turmas avaliadas, dos alunos de instituições particulares, 209 (39,2\%) têm bolsa do Programa Universidade para Todos PROUNI) ou Fundo de Financiamento Estudantil (FIES) . Desses, 129 (61,7\%) encontravam-se no início do curso, 38 (18,2\%) no meio do curso e 42 (20,1\%) no último ano. Entre os estudantes do início do curso que já experimentaram tabaco, 70,9\% residiam com os pais, $17,7 \%$ com amigos e $11,3 \%$ sozinhos ( $\mathrm{p}=0,002)$. Dos estudantes do meio do curso, a maioria $(86,0 \%)$ residia com os pais. Já entre os do final do curso, $87(94,5 \%)$ moravam com os pais e $5(5,4 \%)$ moravam sozinhos.

Não houve diferença significativa entre os estudantes, do início do curso $(p=0,414)$, do meio do curso $(p=0,175)$ ou do final do curso ( $\mathrm{p}=0,416)$, de instituições particulares e públicas em relação ao fato de já terem usado alguma substância derivada do tabaco. Por outro lado, observou-se que entre os 392 alunos dos S1/S2, 62 (15,8\%) já haviam consumido algum derivado do tabaco. Esse consumo foi significativamente maior entre o sexo masculino $(\mathrm{p}=0,025)$, sem diferença em relação a estado civil $(p=0,247)$, renda familiar $(p=0,191)$ ou receber algum tipo de bolsa acadêmica $(p=0,757)$ ou Prouni/ Fies $(\mathrm{p}=0,483)$.

Dos 319 alunos que se encontravam no S7/S8, 100 (31,3\%) já haviam experimentado alguma droga derivada do tabaco. Entre os homens, o percentual de consumo foi significativamente maior ( $p=0,001)$, mas não houve diferença entre o estado civil ( $p=0,677)$.

Dos 324 estudantes no I3/I4, 92 (28,4\%) já haviam experimentado alguma droga derivada do tabaco. Essa experiência se mostrou significativamente superior nos homens, com $44,0 \%$ deles em comparação a $15,1 \%$ das mulheres $(p=0,000)$. No entanto, não foi observada diferença entre os estados civis $(p=0,228)$. 
Entre todos os entrevistados, 11,8\% convivem com algum fumante em casa, sem diferença significativa em relação à origem da instituição de ensino ( $p=0,784)$, e 38,6\% estavam nos S1/S2, 25,2\% estavam cursando os S7/S8, e 36,1\% os I3/I4.

A maior parte dos estudantes do início e do meio do curso afirmou que acadêmicos de Medicina deveriam receber treinamento sobre técnicas de cessação do uso de tabaco, 89,4\% e 88,7\%, respectivamente, independentemente do fato de já terem usado alguma substância derivada do tabaco. Já entre os acadêmicos do final do curso, a maior parcela (59,4\%) afirmou o contrário.

Entre os estudantes do início do curso que experimentaram alguma substância derivada do tabaco, 60 (96,8\%) (p = $0,000)$ já se embriagaram pelo menos uma vez na vida. Dos alunos do meio do curso e nessa mesma condição, 93 (93,0\%) ( $\mathrm{p}=0,000$ ) já se embriagaram pelo menos uma vez na vida. Todos os acadêmicos do final do curso que experimentaram alguma substância derivada do tabaco já ingeriram bebida alcoólica alguma vez na vida $(p=0,016)$.

Dos 392 alunos do início do curso, 329 (83,9\%) já consumiram alguma bebida alcoólica em algum momento da vida, sem diferença significativa entre os sexos $(p=0,229)$, estado civil ( $p=0,247)$, residir em Fortaleza $(p=0,062)$ e origem da instituição de ensino $(\mathrm{p}=0,414)$. Por outro lado, o consumo foi maior entre aqueles estudantes cuja família apresentou renda superior a nove salários mínimos $(\mathrm{p}=0,001)$.

Entre os 319 estudantes do meio do curso, 296 (92,8\%) já ingeriram bebida alcoólica, sem diferença significativa entre sexo ( $p=0,623)$, estado civil $(p=0,321)$ ou ter nascido em Fortaleza $(p=0,454)$. Porém, da mesma forma que nos semestres iniciais, o fato de ter renda familiar mensal maior do que nove salários mínimos esteve associado ao maior consumo de álcool $(p=0,006)$.

A proporção de estudantes de instituições públicas $(19,0 \%)$ que nunca consumiu bebida alcoólica não mostrou diferença estatisticamente significante em relação às instituições privadas $(18,6 \%)(R P=1,00-I C: 0,94-1,06 ; p=0,912)$.

Dos 324 alunos do final do curso, 310 (96,7\%) já consumiram bebida alcoólica em algum momento da vida. Não houve diferença estatisticamente significante entre sexo $(p=0,174)$, estado civil ( $p=0,081)$, ter nascido em Fortaleza $(p=0,148)$, a renda fixa mensal $(p=0,448)$ e a instituição de ensino ( $p$ $=0,444)$. Morar com os pais não foi fator de proteção para o consumo de álcool em nenhum período do curso ( $\mathrm{p}=0,082$; $p=0,316 ; p=0,035$, respectivamente). Apesar da significância estatística nos alunos do internato, a razão de prevalência não foi estatisticamente significativa ( $R P=1,12$ - IC:0,98-1,27).

A maioria dos estudantes que já consumiu bebida alcoólica, tanto do início como do meio do curso (56,2\%; $p=0,000$ e $64,2 \% ; p=0,000)$, afirmou fazer uso de bebidas alcoólicas principalmente nas festas da faculdade $(p=0,000)$. Já entre os acadêmicos do final do curso, a maioria consome bebidas alcoólicas tanto nas festas da faculdade, $62,9 \%(p=0,000)$ como nos finais de semana, 56,7\% $(\mathrm{p}=0,000)$.

A dependência de álcool foi afirmada por apenas seis dos 210 respondentes (2,8\%). Entretanto, dos 116 que responderam à pergunta se já tentaram parar de beber, 27 (23,3\%) afirmaram que sim. Isto está diretamente relacionado ao consumo excessivo de álcool que foi relatado por 836 (83,8\%) alunos.

Tabela 1

Informações relacionadas ao consumo de bebidas alcoólicas por alunos de Medicina das universidades do município de Fortaleza (CE)

\begin{tabular}{|c|c|c|}
\hline Variáveis & № & $\%$ \\
\hline $\begin{array}{l}\text { Consumo atual de bebida alcoólica } \\
\text { Não } \\
\text { Sim }\end{array}$ & $\begin{array}{l}173 \\
747\end{array}$ & $\begin{array}{l}18,80 \\
81,20\end{array}$ \\
\hline $\begin{array}{l}\text { Frequência com que consome bebidas } \\
\text { Ocasionalmente } \\
\text { Pelo menos uma vez por mês } \\
1 \text { ou } 2 \text { vezes por semana } \\
3 \text { ou } 4 \text { vezes por semana } \\
5 \text { vezes por semana ou mais }\end{array}$ & $\begin{array}{r}414 \\
200 \\
184 \\
6 \\
1\end{array}$ & $\begin{array}{r}51,43 \\
24,84 \\
22,86 \\
0,75 \\
0,12\end{array}$ \\
\hline $\begin{array}{l}\text { Já se embriagou } \\
\text { Não } \\
\text { Sim }\end{array}$ & $\begin{array}{l}266 \\
656\end{array}$ & $\begin{array}{l}28,85 \\
71,15\end{array}$ \\
\hline $\begin{array}{l}\text { Principal tipo de bebida } \\
\text { Whisky } \\
\text { Vodca } \\
\text { Vinho } \\
\text { Cerveja/chope } \\
\text { Aguardente/cachaça } \\
\text { Rum } \\
\text { Outro }\end{array}$ & $\begin{array}{r}41 \\
243 \\
58 \\
356 \\
14 \\
2 \\
13\end{array}$ & $\begin{array}{r}5,64 \\
33,43 \\
7,98 \\
48,97 \\
1,93 \\
0,28 \\
1,79\end{array}$ \\
\hline $\begin{array}{l}\text { Com que frequência exagerou o consumo } \\
\text { Nunca } \\
\text { Ocasionalmente } \\
\text { Mensalmente } \\
\text { Mais de uma vez no mês } \\
\text { Semanalmente }\end{array}$ & $\begin{array}{r}291 \\
529 \\
43 \\
13 \\
9\end{array}$ & $\begin{array}{r}32,88 \\
59,77 \\
4,86 \\
1,47 \\
1,02\end{array}$ \\
\hline $\begin{array}{l}\text { Qual o período em que era maior o consumo de } \\
\text { bebida } \\
\text { Antes de preparar-se para o vestibular } \\
\text { Ao estudar para o vestibular } \\
\text { Logo que entrou na faculdade } \\
\text { Durante o período intermediário da faculdade } \\
\text { Durante o período do internato }\end{array}$ & $\begin{array}{r}105 \\
92 \\
339 \\
191 \\
48\end{array}$ & $\begin{array}{r}13,55 \\
11,87 \\
43,74 \\
24,65 \\
6,19\end{array}$ \\
\hline $\begin{array}{l}\text { Houve mudança de hábito de beber após entrar na } \\
\text { faculdade } \\
\text { Não, não bebia e continuo sem beber } \\
\text { Não, continuo bebendo a mesma quantidade } \\
\text { Sim, aumentei o consumo } \\
\text { Sim, diminuí o consumo }\end{array}$ & $\begin{array}{l}113 \\
270 \\
375 \\
120\end{array}$ & $\begin{array}{l}12,87 \\
30,75 \\
42,71 \\
13,67\end{array}$ \\
\hline
\end{tabular}


O recebimento de informações sobre consumo exagerado de álcool e suas consequências foi relatado por 83,8\% dos alunos. No entanto, 91,8\% deles afirmaram que ainda é necessário um treinamento específico para cessar o uso continuado de álcool. Apenas 39,5\% afirmaram estar aptos a aconselhar um paciente a não ingerir bebidas alcoólicas; os demais não se consideram aptos ou não souberam responder a esta pergunta, uma vez que apenas $28,4 \%$ receberam algum treinamento neste sentido na grade curricular de sua universidade.

Conforme a Tabela 1, o consumo de bebida ainda está presente em mais de $80 \%$ dos alunos entrevistados e este consumo se deu pelo menos uma vez no último mês. A presença de embriaguez é maior que $70 \%$, sendo a cerveja e a vodca as bebidas mais utilizadas. Destaca-se que o período de maior consumo durante a vida acadêmica se deu logo no início da faculdade em aproximadamente 43\% (339 estudantes). Isso pode ser corroborado pelo fato de $42,7 \%$ dos alunos terem referido que aumentaram o consumo de bebida alcoólica após o início da faculdade.

Associando o consumo de álcool e ser tabagista, pode-se observar que em todos os tabagistas há uma idade média e mediana inferior para o consumo de álcool com valores estatisticamente significantes (Tabela 2).

O consumo de tabaco se mostrou de forma diferenciada nos três períodos, sendo significativamente maior $(p<0,001)$ no quarto e sexto ano. A influência de outras pessoas não foi motivo diferencial entre os semestres, bem como fumar em ambiente acadêmico, que se mostrou de forma uniforme nos três períodos avaliados, apesar de ter uma frequência maior entre aqueles do início do curso (Tabela 3).

\begin{tabular}{|c|c|c|c|c|c|c|}
\hline \multicolumn{7}{|c|}{$\begin{array}{l}\text { Associação entre ser tabagista e idade, idade em } \\
\text { que experimentou bebida pela primeira vez, idade } \\
\text { da primeira embriaguez e número de doses que } \\
\text { costuma ingerir quando faz uso de bebida alcoólica }\end{array}$} \\
\hline Variáveis contínuas & № & Média & P25 & Mediana & P75 & p-valor \\
\hline $\begin{array}{l}\text { Idade } \\
\text { Tabagismo } \\
\text { Não tabagismo }\end{array}$ & $\begin{array}{l}236 \\
739\end{array}$ & $\begin{array}{l}23,7 \\
22,6\end{array}$ & $\begin{array}{l}22,0 \\
20,0\end{array}$ & $\begin{array}{l}23,6 \\
22,4\end{array}$ & $\begin{array}{l}25,3 \\
24,5\end{array}$ & 0,000 \\
\hline $\begin{array}{l}\text { Idade em que experiment } \\
\text { bebida } \\
\text { Tabagismo } \\
\text { Não tabagismo }\end{array}$ & $\begin{array}{l}250 \\
664\end{array}$ & $\begin{array}{l}14,8 \\
16,6\end{array}$ & $\begin{array}{l}14 \\
15\end{array}$ & $\begin{array}{l}15 \\
16\end{array}$ & $\begin{array}{l}16 \\
18\end{array}$ & 0,0000 \\
\hline $\begin{array}{l}\text { Idade em que se embriag } \\
\text { pela primeira vez } \\
\text { Tabagismo } \\
\text { Não tabagismo }\end{array}$ & $\begin{array}{l}241 \\
402\end{array}$ & $\begin{array}{l}16,3 \\
18,2\end{array}$ & $\begin{array}{l}15 \\
17\end{array}$ & $\begin{array}{l}16 \\
18\end{array}$ & $\begin{array}{l}18 \\
20\end{array}$ & 0,0000 \\
\hline $\begin{array}{l}\text { Número de doses } \\
\text { Tabagismo } \\
\text { Não tabagismo }\end{array}$ & $\begin{array}{l}230 \\
526\end{array}$ & $\begin{array}{l}5,5 \\
3,3\end{array}$ & $\begin{array}{l}3 \\
2\end{array}$ & $\begin{array}{l}5 \\
3\end{array}$ & $\begin{array}{l}6 \\
4\end{array}$ & 0,0000 \\
\hline
\end{tabular}

TABELA 3

Associação entre características de consumo de tabaco e período do curso de Medicina em Fortaleza (CE)

\begin{tabular}{|c|c|c|c|c|c|c|}
\hline Semestres & Total & $\mathbf{N}$ & $\%$ & $\mathbf{R P}$ & IC & P valor \\
\hline \multicolumn{7}{|c|}{ Utilizou tabaco } \\
\hline S1/S2 & 392 & 62 & 15,8 & 1 & - & \multirow{3}{*}{$<0,001$} \\
\hline S7/S8 & 319 & 100 & 31,3 & 1,98 & $1,49-2,62$ & \\
\hline $\mathrm{I} 3 / \mathrm{I} 4$ & 322 & 92 & 28,6 & 1,80 & $1,35-2,40$ & \\
\hline \multicolumn{7}{|c|}{ Presença de outro fumante em casa } \\
\hline S1/S2 & 339 & 48 & 14,1 & 1 & - & \multirow{3}{*}{0,353} \\
\hline S7/S8 & 286 & 31 & 10,8 & 0,76 & $0,50-1,16$ & \\
\hline $\mathrm{I} 3 / \mathrm{I} 4$ & 297 & 43 & 14,5 & 1,02 & $0,69-1,49$ & \\
\hline \multicolumn{7}{|c|}{ Começou a fumar por influência de outros } \\
\hline S1/S2 & 46 & 25 & 54,3 & 1 & - & \multirow{3}{*}{0,389} \\
\hline S7/S8 & 75 & 35 & 46,7 & 1,33 & $0,89-2,00$ & \\
\hline $\mathrm{I} 3 / \mathrm{I} 4$ & 59 & 24 & 40,7 & 1,14 & $0,77-1,69$ & \\
\hline \multicolumn{7}{|c|}{ Foi criticado por fumar } \\
\hline S1/S2 & 283 & 134 & 47,3 & 1 & - & \multirow{3}{*}{0,028} \\
\hline S7/S8 & 284 & 107 & 37,7 & 0,79 & $0,65-0,96$ & \\
\hline $\mathrm{I} 3 / \mathrm{I} 4$ & 290 & 110 & 37,9 & 0,80 & $0,66-0,97$ & \\
\hline \multicolumn{7}{|c|}{ Fuma na universidade } \\
\hline S1/S2 & 36 & 2 & 5,5 & 1 & - & \multirow{3}{*}{0,352} \\
\hline S7/S8 & 68 & 1 & 1,5 & 0,26 & $0,02-2,82$ & \\
\hline $\mathrm{I} 3 / \mathrm{I} 4$ & 63 & 1 & 1,6 & 0,28 & $0,02-3,04$ & \\
\hline
\end{tabular}

Fumar em ambiente hospitalar

$\begin{array}{lllll}\text { S1/S2 } & 32 & 0 & 0,0 & 1 \\ \text { S7/S8 } & 69 & 2 & 2,9 & - \\ \text { I3/I4 } & 61 & 2 & 3,9 & -\end{array}$

$0,834^{*}$

Estudantes deveriam receber treinamento para parar de fumar (população total)

$\begin{array}{lllllcc}\text { S1/S2 } & 368 & 329 & 89,4 & 1 & - & \\ \text { S7/S8 } & 294 & 261 & 88,7 & 0,99 & 0,94-1,04 & 0,924 \\ \text { I3/I4 } & 303 & 272 & 89,7 & 1,00 & 0,95-1,05 & \end{array}$

Estudantes deveriam receber treinamento para parar de fumar (apenas fumantes)

\begin{tabular}{lcrrlcc} 
S1/S2 & 58 & 45 & 77,5 & 1 & - & \\
S7/S8 & 93 & 81 & 87,1 & 1,12 & $0,95-1,31$ & 0,139 \\
I3/I4 & 90 & 80 & 88,9 & 1,14 & $0,98-1,33$ & \\
\multicolumn{5}{l}{ Tentou parar de fumar } \\
S1/S2 & 29 & 7 & 24,1 & 1 & - & \\
S7/S8 & 49 & 9 & 18,4 & 0,76 & $0,31-1,82$ & 0,483 \\
I3/I4 & 38 & 11 & 28,9 & 1,19 & $0,53-2,71$ &
\end{tabular}

* Exato de Fischer.

Apenas quatro estudantes fazem uso de tabaco em ambiente hospitalar, nenhum dos semestres iniciais, não havendo diferença significativa entre os semestres (Tabela 3).

Todos os alunos, uniformemente, afirmam a necessidade de receber treinamento para parar de fumar, mas, se fizermos um corte apenas para aqueles que fazem uso de tabaco, a proporção aumenta com o semestre de estudo. 
TABELA 4

Associação entre características de consumo de álcool

e período do curso de Medicina em Fortaleza (CE)

\begin{tabular}{lcccccc}
$\begin{array}{l}\text { Semestre } \\
\text { Consumiu bebida }\end{array}$ & Total & N & \% & RP & IC & p-valor \\
S1/S2 & 392 & 329 & 83,9 & 1 & - & \\
S7/S8 & 318 & 296 & 93,0 & 1,10 & $1,05-1,16$ & $<0,001$ \\
I3/I4 & 324 & 310 & 95,7 & 1,14 & $1,08-1,19$ & \\
\multicolumn{5}{l}{ Faz consumo de bebida } \\
S1/S2 & 325 & 242 & 74,4 & 1 & - & \\
S7/S8 & 289 & 242 & 83,7 & 1,12 & $1,03-1,22$ & $<0,001$ \\
I3/I4 & 306 & 263 & 85,9 & 1,15 & $1,06-1,24$ &
\end{tabular}

Já se embriagou em festas da faculdade

$\begin{array}{lllllcl}\text { S1/S2 } & 392 & 185 & 47,2 & 1 & - & \\ \text { S7/S8 } & 319 & 190 & 59,5 & 1,26 & 1,09-1,45 & <0,001 \\ \text { I3/I4 } & 324 & 195 & 60,2 & 1,27 & 1,11-1,46 & \end{array}$

Já se embriagou em festas da família

$\begin{array}{lllllcc}\text { S1/S2 } & 392 & 83 & 21,2 & 1 & - & \\ \text { S7/S8 } & 319 & 85 & 26,6 & 1,25 & 0,96-1,63 & 0,088 \\ \text { I3/I4 } & 324 & 90 & 27,8 & 1,31 & 1,01-1,70 & \end{array}$

Já se embriagou depois de uma prova

$\begin{array}{lllllcc}\text { S1/S2 } & 392 & 73 & 18,6 & 1 & - & \\ \text { S7/S8 } & 319 & 72 & 22,6 & 1,21 & 0,90-1,61 & 0,017 \\ \text { I3/I4 } & 324 & 45 & 13,9 & 0,74 & 0,53-1,04 & \end{array}$

Já se embriagou no final de semana

$\begin{array}{lllllcl}\text { S1/S2 } & 392 & 121 & 30,9 & 1 & - & \\ \text { S7/S8 } & 319 & 143 & 44,8 & 1,45 & 1,20-1,75 & <0,001 \\ \text { I3/I4 } & 324 & 176 & 54,3 & 1,75 & 1,47-2,10 & \end{array}$

Considera-se dependente de álcool

$\begin{array}{lllllcc}\text { S1/S2 } & 57 & 2 & 3,5 & 1 & - & \\ \text { S7/S8 } & 81 & 3 & 3,7 & 1,05 & 0,18-6,11 & 0,669 \\ \text { I3/I4 } & 72 & 1 & 1,4 & 0,39 & 0,03-4,25 & \end{array}$

Estudantes devem receber treinamento específico sobre como desestimular o consumo excessivo de álcool

$\begin{array}{lllllcc}\text { S1/S2 } & 387 & 353 & 91,2 & 1 & - & \\ \text { S7/S8 } & 317 & 290 & 91,5 & 1,00 & 0,95-1,04 & 0,713 \\ \text { I3/I4 } & 321 & 298 & 92,8 & 1,01 & 0,97-1,06 & \end{array}$

Recebeu esse treinamento na grade curricular

$\begin{array}{lrrrlcl}\text { S1/S2 } & 379 & 42 & 11,1 & 1 & - & \\ \text { S7/S8 } & 314 & 91 & 29,0 & 2,61 & 1,87-3,65 & <0,001 \\ \text { I3/I4 } & 322 & 155 & 48,1 & 4,34 & 3,19-5,90 & \end{array}$

Durante as aulas, recebeu informações sobre os malefícios do excesso de álcool

$\begin{array}{lllllll}\text { S1/S2 } & 370 & 279 & 75,4 & 1 & - & \\ \text { S7/S8 } & 309 & 272 & 88,0 & 1,16 & 1,08-1,25 & <0,001 \\ \text { I3/I4 } & 318 & 285 & 89,6 & 1,18 & 1,10-1,27 & \end{array}$

Sente-se apto a aconselhar um paciente a parar de beber

\begin{tabular}{lrrrrrr} 
S1/S2 & 169 & 76 & 44,9 & 1 & - & \\
S7/S8 & 198 & 119 & 60,1 & 1,33 & $1,09-1,63$ & $<0,001$ \\
I3/I4 & 235 & 169 & 71,9 & 1,59 & $1,32-1,92$ & \\
\hline
\end{tabular}

Outro ponto que merece destaque é a proporção de alunos que tentou parar de fumar. De forma geral, menos de $25 \%$ já tentou, e a maior proporção se deu nos alunos do internato com 28,9\% (Tabela 3).

A proporção de estudantes que consumiu bebidas alcoólicas é muito superior à de tabaco, com a média de 90,8\%. Essa proporção foi diferenciada e crescente entre os semestres, com maior prevalência entre os alunos do internato $(95,7 \%)$. $\mathrm{O}$ mesmo padrão foi observado entre aqueles que fazem consumo atual de bebidas alcoólicas, com diferença significante estatisticamente $(\mathrm{p}<0,001)$ entre os três períodos avaliados; da mesma forma, o internato mostrou maior prevalência, $14 \%$ maior que os alunos do início do curso.

A embriaguez é sempre presente entre todos os semestres, sendo muito frequente em festas da faculdade, de forma semelhante nos semestres intermediários e finais do curso. Comparativamente, em festas familiares, a proporção é bem mais reduzida, sem diferença significativa entre os semestres.

Os momentos mais propícios ao consumo exagerado de álcool são depois de uma avaliação ou no final de semana. $\mathrm{O}$ primeiro se mostrou mais frequente nos alunos do período intermediário, com 22,6\% de prevalência, enquanto o final de semana foi mais frequente nos alunos do internato, apesar de haver um crescimento gradual das proporções.

A autopercepção como dependente do álcool foi muito reduzida, com apenas seis indivíduos nos três períodos avaliados. Entretanto, mais de $90 \%$, em todos os períodos, afirmam que devem receber treinamento de forma específica para desestimular o consumo excessivo de álcool, e este treinamento foi dado na grade curricular apenas para $11,1 \%$ no início do curso, chegando a 48,1\% no final. Por outro lado, mais de $75 \%$ deles receberam informações sobre os malefícios do álcool durante as aulas. A maior proporção de alunos que recebeu estas informações pode estar diretamente ligada a se sentir apta a realizar aconselhamento a um paciente, uma vez que essa proporção aumenta significativamente com o semestre avaliado.

Algo relevante é o fato de que 59,5\% dos alunos do início do curso já haviam se embriagado até o momento da pesquisa. Este percentual foi superior nos dois outros períodos da faculdade, com valores maiores que $75 \%$ e significativamente superiores (Tabela 5).

A idade em que os alunos se embriagaram pela primeira vez é praticamente a mesma em todos os semestres: 17 anos para os semestres iniciais e intermediários, e 18 para o internato. No entanto, esta diferença foi estatisticamente significante ( $p<0,001)$. Os intervalos interquartis foram de 16 a 18, 15 a 19, e 16 a 20 anos, respectivamente. 


\begin{tabular}{ccccccc} 
TABELA 5 \\
Proporção de alunos de Medicina de Fortaleza \\
(CE) que já se embriagou alguma vez na vida \\
de acordo com os semestres cursados \\
Semestre & Total & N & $\%$ & RP & IC & p-valor \\
S1/S2 & 321 & 191 & 59,5 & 1 & - & \\
S7/S8 & 294 & 226 & 76,8 & 1,29 & $1,15-1,44$ & $<0,001$ \\
I3/I4 & 307 & 239 & 77,8 & 1,31 & $1,17-1,45$ & \\
\hline
\end{tabular}

\section{DISCUSSÃO}

O consumo de álcool foi muito elevado em nossa casuística. Isso preocupa principalmente por ser uma droga socialmente aceita. O consumo de álcool e de outras drogas está presente de forma importante na comunidade médica e frequentemente esse consumo se inicia durante a faculdade. A prevalência do consumo de álcool encontrada foi acima de $80 \%$.

Um trabalho realizado em mais de cem cidades brasileiras com mais de 200 mil habitantes apontou que o consumo de álcool é muito relevante na faixa etária de 18 a 24 anos e que $15,5 \%$ dessa população referem dependência ${ }^{17}$. Entre estudantes de Medicina, esse percentual variou de $25 \%$ de consumo exagerado até $98 \%$ em algumas regiões, dependendo da amostra e da forma de coleta dos dados ${ }^{18-20}$. Além da elevada prevalência de consumo, há uma preocupação importante com o uso exagerado de álcool e embriaguez. Estudantes de Juiz de Fora, por exemplo, apresentaram prevalência de $25 \%$ de consumo exagerado ${ }^{18}$. Essa elevada ingesta alcoólica está relacionada ao consumo associado do tabagismo e principalmente ao sexo masculino. Por outro lado, ter religião e relacionamento fixo atuou como protetor desse consumo. Entre estudantes de Minas Gerais, a prevalência do consumo de álcool foi de $60 \%$, e $25 \%$ destes referiram a necessidade de buscar programas para prevenir danos causados pelo consumo abusivo de bebida alcoólica ${ }^{19}$.

Apesar da alta prevalência de experimentação de álcool e até mesmo de embriaguez, registrada em todos os períodos avaliados, a dependência de álcool referida pelos estudantes foi muito baixa, independentemente do período avaliado. Em outras regiões, esse percentual chegou a $25 \%{ }^{18}$. É previsível esse achado se considerarmos que alunos com dependência de álcool teriam muita dificuldade em avançar na faculdade e até mesmo em chegar aos semestres finais, já que os prejuízos causados pela dependência de álcool limitariam grande parte das atividades acadêmicas e profissionais.

Estudos apontam que o consumo de álcool mais pesado esteve associado à realização de festas na faculdade, a semestres mais avançados e ao fato de não residir com os pais, perfil muito semelhante ao relatado em outra cidade no Nordeste do Brasil ${ }^{21}$. Esses aspectos apresentam tendência crescente em virtude da forma de seleção dos alunos, que aumenta o número de estudantes de Medicina estudando em cidades distintas das de seus familiares. É necessário orientá-los sobre os riscos do consumo excessivo de álcool e pensar em alternativas para trabalhar com esses estudantes de forma que não tenhamos um aumento ainda mais significativo do consumo dessas drogas e principalmente de possíveis consequências que este hábito pode trazer para sua profissão.

Os alunos entrevistados neste trabalho julgaram importante a incorporação de treinamentos sobre os prejuízos provenientes do consumo excessivo de álcool durante sua formação acadêmica. Mas um estudo recente realizado na região amazônica, por exemplo, aponta limitações importantes na formação universitária dos profissionais de saúde para trabalhar com pessoas que têm uso problemático de álcool ${ }^{22}$.

A juventude brasileira ${ }^{23}$ tem cultivado o hábito de se embriagar. No Brasil, estudos epidemiológicos mostram que o consumo de álcool é maior entre universitários do que entre estudantes do ensino médio. Esses índices são preocupantes, pois no futuro podem acarretar problemas de saúde mais graves e a própria dependência, já que esses jovens têm contato com a droga cada vez mais cedo.

Estima-se que o uso de álcool esteja aumentando em decorrência do estilo de vida, ansiedade, estresse, depressão e baixa autoestima9. Os universitários estão mais expostos aos ambientes em que o álcool é mais fácil. Isso aponta uma necessidade maior de o ambiente acadêmico oferecer apoio e treinamento efetivo para esses profissionais/estudantes pararem de beber.

Desde a década passada já apontava que os hábitos desenvolvidos durante a vida universitária poderiam ser levados para a vida profissional ${ }^{24}$. Tal comportamento pode interferir no aumento dos índices de médicos dependentes de álcool, interferindo, assim, em seu ambiente de trabalho. Naturalmente, quando um estudante de Medicina ou profissional de saúde assume a dependência alcoólica, isso ocorre numa fase mais tardia, tornando mais difícil o início de um tratamento.

Apesar de considerada baixa essa dependência, os dados mostram que o consumo vem crescendo a cada dia. A preocupação, além da dependência alcoólica, é com a associação com outras drogas, como o tabaco. Em São Paulo, a prevalência do uso de drogas entre estudantes de Medicina é alta, destacando-se o álcool, mas já aparecendo tabaco, maconha, solventes e tranquilizantes ${ }^{20}$. Em nosso estudo, percebemos uma redução no consumo de cigarro quando comparado a estudos realizados anteriormente. Estudantes de Medicina da cidade 
de Uberlândia, por exemplo, apresentavam perfil semelhante desde a década passada ${ }^{20}$. Esses achados sugerem uma consciência maior sobre os malefícios do cigarro, mas o mesmo não ocorre com o consumo abusivo de bebidas alcoólicas, em que se observa um comportamento de risco.

Os estudantes e profissionais da área da Saúde têm papel de destaque na sensibilização da população para a redução do hábito tabagista ${ }^{25}$. Entretanto, o período acadêmico é considerado propício ao início do hábito de fumar por aspectos associados, entre outros fatores, ao ambiente universitário ${ }^{26}$. Além disso, tal situação preocupa pelo fato de que, quanto mais precoce for a experimentação do tabaco, maior será o risco de dependência da nicotina.

O tabagismo permanece como uma das principais causas de enfermidade evitáveis e o seu consumo ainda é alarmante em alguns grupos. Mesmo que os estudantes tenham conhecimento sobre os prejuízos para a saúde, quanto maior o uso e a idade mais avançada, mais difícil se tornará a cessação.

Vontade própria e influência dos amigos foram referidas pela maior parte dos estudantes como justificativa para iniciar o consumo de cigarros. E tanto o hábito de fumar como o número de cigarros fumados por dia foram maiores entre os estudantes do internato, provavelmente por ser o momento em que se encontram mais tensos e com acúmulo de funções. A maioria dos alunos entrevistados afirma que um treinamento para parar de fumar é necessário para os estudantes de Medicina.

\section{CONCLUSÃO}

A prevalência do consumo de álcool entre os estudantes de Medicina é muito elevada, principalmente entre aqueles que relataram ter fumado alguma vez na vida. O consumo de álcool e a formação sobre a cessação do hábito tabagista foram abordados de forma incipiente durante a formação acadêmica, independentemente da instituição. É preciso reforçar esses aspectos na formação desses futuros profissionais de saúde.

\section{REFERÊNCIAS}

1. Peto R, Lopez AD, Boreham J, Thun M, Health Jr C. Mortality from smoking in developed countries: Indirect estimates from National Vital Statistics, 1950-2000. Am J Epidemiol. 1996; 143(5):529-30.

2. Tuyns AJ. Alcohol and cancer. Proceedings of the Nutrition Society. London, 1990; 49: 145-151.

3. Rondina RC, Gorayeb R, Botelho C, Silva AMC. A relação entre tabagismo e características sociodemográficas em universitários. Psicologia Saúde \&Doenças. 2005; 6(1): 35-45.

4. Jemal A, Bray F, Center MM, Ferlay J, Ward E, Forman D. Global cancer statistics. CA Cancer J Clin [Internet].
2011 [capturado em: 10 fev. 2012]; 61(2): 69-90. Disponívelem: <http://onlinelibrary.wiley.com/doi/10.3322/ caac. $20107 / \mathrm{pdf}>$.

5. Schutze M, Boeing H, Pischon T, Rehm J, Kehoe T, Gmel G, et al. Alcohol attributable burden of incidence of cancer in eight European countries based on results from prospective cohort study. BMJ [Internet]. 2011[capturado em: 10 fev. 2012]; 342: d1584. Disponível em: <http:/ /www.bmj. com/highwire/filestream/352620/field_highwire_article_pdf/0.pdf $>$.

6. World Health Organization. Tobacco or health.A global status report.Genebra: WHO, 1997.

7. Mesquita EM, Nunes AJ, Cohen C. Avaliação das atitudes dos estudantes de medicina frente ao abuso de drogas por colegas do meio acadêmico. Revpsiquiatr clín. 2008; 35(supl1): 8-12.

8. Coelho IZ. Experiências discriminatórias e suas relações com consumo de álcool em estudantes universitários do Sul do Brasil. Florianópolis; 2013.Mestrado [Dissertação] -Universidade Federal de Santa Catarina.

9. Feijão IEP, Sampaio HAC, Sabry MOD, Carioca AAF, Yum MEM, Lima JWO. Prática de binge alcoólico entre estudantes universitários. RevBrasPromSaúde. 2012; 25(4):462-468.

10. Menezes AMB, Hallial PC, Silva F, Souza M, Paiva L, D'ávila A, et al. Tabagismo em estudantes de medicina: tendências temporais e fatores associados. J. bras. pneumol. 2004 Maio-Jun; 30(3): 223-8. doi: http://dx.doi. org/10.1590/S1806-37132004000300007.

11. Mattos MHO, Silva LA, Franken RA. Tabagismo no currículo da Faculdade de Ciências Médicas da Santa Casa de São Paulo. Rev. bras. educ. med. 2009; 33(1):33-39.

12. Brasil. Ministério da Saúde. Secretaria de Vigilância em Saúde. Secretaria de Atenção à Saúde. Instituto Nacional de Câncer. Coordenação de Prevenção e Vigilância. Inquérito domiciliar sobre comportamentos de risco e morbidade referida de doenças e agravos não transmissíveis: Brasil, 15 capitais e Distrito Federal, 2002-2003[Internet]. Rio de Janeiro: INCA; 2004 [capturado em: 5 jan. 2012]. Disponível em: <http://www.inca.gov.br/inquerito/docs/ completa.pdf $>$.

13. Smith DR,Leggat PA. An international review of tobacco smoking among medical students. J Postgraduate Med, Mumbai. 2007; 53(1):55-62.

14. Saloojee Y, Steyn K. Educating medical students about tobacco. SAMJ. 2005; 95(5):330-331.

15. Nerin I, Guillén D, Mas A, Crucelaequi A. Evaluation of the influence of medical education on the smoking attitudes of the future doctors. Arch Bronconeumol. 2004;40(8): 341-7. 
16. Richmond R. The process of introducing a tobacco curriculum in medical school. Respirology. 2004; 9(2):165-72.

17. Ramis TR, Mielke GI, Habeyche EC, Oliz MM, Azevedo R, Halla PC. Tabagismo e consumo de álcool em estudantes universitários: prevalência e fatores associados. RevBrasEpidemiol. 2012; 15(2):376-85.

18. Carneiro, EB, Braga RT, Silva FD, Nogueira MC. Fatores Associados a Beber Pesado Episódico entre Estudantes de Medicina. Rev. bras. educ. med. 2012; 36(4):524-530;2012.

19. Rocha LA, Lopes ACFMM, Martelli DRB, Lima VB, Martelli-Júnior H. Consumo de Álcool entre Estudantes de Faculdades de Medicina de Minas Gerais, Brasil. Rev. bras. educ. med. 2011; 35(3):369-375.

20. Paduani GF, Barbosa GA, Morais JCR, Pereira JCP, Prado MFAMM, Almeida NBC, et al. Consumo de álcool e fumo entre os estudantes da Faculdade de Medicina da Universidade Federal de Uberlândia. Rev. bras. educ. med. 2008; 32 (1): $66-75$.

21. Barbosa FL, Barbosa, RL, Barbosa MCL, Aguiar DL, Figueiredo IA, Ribeiro AC, et al. Uso de Álcool entre Estudantes de Medicina da Universidade Federal do Maranhão. Rev. bras. educ. med. 2013; 37(1): 89-95.

22. Moretti-Pires RO, Corradi-Webster CM, Furtado EF. Consumo de Álcool e Atenção Primária no Interior da Amazônia: sobre a Formação de Médicos e Enfermeiros para Assistência Integral. Rev. bras. educ. med. 2011; 35(2):219-228.

23. Amorim AVC, Kikko EO, Abrantes MM, Andrade VLA. Álcool e alcoolismo: estudo de prevalência entre discentes do curso de Medicina da UNIFENAS em Belo Horizonte - Minas Gerais. Revista Médica de Minas Gerais. 2008;18(1):16-23.

24. Martinho AF, Tonin CL, Nunes LM, Novo NF, Hubner CVK. Uso de álcool e drogas por acadêmicos dos cursos de enfermagem, biologia e medicina na pontífica universidade católica de São Paulo. Rev. Fac. Ciênc. Méd. Sorocaba. 2009;11(1):11-15.
25. Magliari RT, Pagliusi AL, Previero BM, Menezes FR, Feldman A, Novo NF. Prevalência de tabagismo em estudantes de faculdade de medicina. RevMed (São Paulo). 2008;87(4):264-71.

26. Manzano BM, Ramos EMC, Vanderei LCM, Ramos D. Tabagismo no ambiente universitário: grau de dependência, sintomas respiratórios e função pulmonar. Arq. Ciênc. Saúde UNIPAR. 2009;13(2):75-80.

\section{AGRADECIMENTOS}

Os alunos receberam bolsa de pesquisa (iniciação científica) do Centro Universitário Unichristus para a realização das atividades relacionadas a este trabalho.

\section{CONTRIBUIÇÃO DOS AUTORES}

Marcelo de Almeida Pinheiro, Levi Freitas Torres, Matheus Sales Bezerra, Rodrigo Cardoso Cavalcante, Raquel Diógenes Alencar, Amanda Carneiro Donato e Camila Pontes Bessa Campêlo participaram do desenho do estudo, coleta de dados, análise dos dados, redação do artigo e revisão da versão final. Marcelo de Almeida Pinheiro, Ileana Pitombeira Gomes, Carlos Henrique Morais de Alencar e Luciano Pamplona de Góes Cavalcanti participaram igualmente do desenho do estudo, da análise estatística dos dados, redação do artigo, discussão dos resultados e revisão da versão final.

\section{CONFLITO DE INTERESSES}

Os autores declaram não haver conflito de interesses.

\section{ENDEREÇO PARA CORRESPONDÊNCIA}

Luciano Pamplona de Góes Cavalcanti

Faculdade de Medicina da Universidade Federal do Ceará

Departamento de Saúde Comunitária

Rua Prof. Costa Mendes, 1608, 5andar - Fortaleza

CEP 60430-140 - CE

E-mail: pamplona.luciano@gmail.com 